\title{
Channeling radiation as a source of hard x-rays with high spectral brilliance
}

\author{
C. A. Brau ${ }^{1}$, B.-K. Choi ${ }^{1}$, J. D. Jarvis ${ }^{1}$, J. W. Lewellen ${ }^{2}$, and P. Piot ${ }^{3,4}$ \\ ${ }^{1}$ Vanderbilt University, Nashville, TN 37235 \\ ${ }^{2}$ Naval Postgraduate School, Monterey, CA 93943 \\ ${ }^{3}$ Northern Illinois University, DeKalb, IL 60115 \\ ${ }^{4}$ Fermi National Accelerator Laboratory, Batavia, IL 60510
}

Starting with Bragg's introduction of crystallography in 1912, Moseley’s ordering of the chemical elements in 1913, and Compton's discovery of the momentum of a photon, much of what we've learned about the physical world in the last century has been learned using x-rays. $\mathrm{X}$-rays are now an indispensable tool in materials science and protein crystallography. In medicine, too, we’ve progressed from Roentgen's 1895 shadow images of the bones in his hand to 3-D computerized tomography.

Paralleling - and enabling - the advances in x-ray applications has been the continued development of x-ray sources, which have moved from Roentgen's primitive Crookes tube to the recent development of the $x$-ray free-electron laser (FEL) at SLAC. Unfortunately, the hard xray FEL is so large and expensive that there's only one in the world (soon to be two), and an experiment requiring one day on the machine costs about \$1M just for the machine time. Your dentist won't have one any time soon. For this reason, most of the world's needs for x-rays are satisfied by a variety of "conventional” sources and a growing number of unconventional sources that are smaller, cheaper, and can be dispersed among the labs and offices where the x-rays are used.

To compare these sources, it is useful to introduce a figure of merit. Although the total number of x-ray photons is a useful measure for many applications, such as sterilizing food, newer applications in medical imaging and physics research depend on the coherence of the x-rays. This is better represented by the spectral brilliance of the x-ray beam, which is defined as the number of photons per second, per unit area, per unit solid angle, per unit relative bandwidth:

$$
B_{v}=\frac{v d^{4} n}{d A d \Omega d v d t}
$$

where $n$ is the number of photons, $v$ the frequency, $t$ the time, $A$ the area, and $\Omega$ the solid angle of the beam. The importance of the spectral brilliance can be illustrated by focusing the $x$ ray beam on a specimen using a zone plate. Compared to the flux at the zone plate, the flux (photons per unit area per unit time) at the focus is increased by the factor $4 N^{2}$, where $N$ is the number of zones. But, the number of zones cannot usefully exceed the reciprocal bandwidth of the $\mathrm{x}$-ray beam or coherence of the interfering waves is lost. Putting this together with some simple geometric arguments, we find that the flux at the focus is on the order of $\Phi \sim \lambda B_{v} / 2 f$, where $\lambda$ is the wavelength of the x-rays and $f$ is the focal length of the zone plate. This depends only on the spectral brilliance. In a modern synchrotron with a spectral brilliance on the order of $10^{19}$ photons $/ \mathrm{s} / \mathrm{mm}^{2} / \mathrm{mrad}^{2} / 0.1 \%$ BW at a wavelength of $1 \mathrm{~nm}(1.2 \mathrm{keV})$, and a focal 
length of $1 \mathrm{~cm}$, the photon flux at the focus is $10^{27}$ photons $/ \mathrm{m}^{2}$-s. For a dipole-allowed, lifetimebroadened transition, the cross section is on the order of $\lambda^{2} / 4 \sim 10^{-19} \mathrm{~m}^{2}$, so the rate of excitation of each atom in the sample is $10^{8} / \mathrm{s}$ ! This makes it possible to detect a single atom in fluorescence.

Conventional x-ray sources are the result of a century's worth of engineering improvements to the original Crookes tube used by Roentgen. Electrons are accelerated electrostatically to about 10-100 kV and impact a metal target. The x-radiation consists of sharp lines (a few eV wide) from inner-shell transitions in the atoms of the anode plus a broad Bremsstrahlung background. The principle limitation in conventional $\mathrm{x}$-ray tubes is heating of the anode by electron impact. To address this problem, high-power tubes use a rotating anode, and even liquid-metal jets have been used. The spectral brilliance from a high-power tube can be as high as $10^{10}$ photons/s-mm ${ }^{2}$ $\mathrm{mrad}^{2}-0.1 \% \mathrm{BW}$ at the characteristic lines of the anode, such as $9 \mathrm{keV}$ for $\mathrm{Cu} \mathrm{K}_{\alpha}$ and $20 \mathrm{keV}$ for Mo $\mathrm{K}_{\alpha}$ radiation. The Bremsstrahlung background is broad and accounts for most of the photons that are radiated, but the spectral brilliance of the Bremsstrahlung is about three orders of magnitude smaller.

Synchrotron radiation sources presently offer the highest spectral brilliance in the x-ray regime. Broadband radiation is generated in the bend magnets of an electron storage ring. Spectral brilliance as high as $10^{15}$ photons $/ \mathrm{s} / \mathrm{mm}^{2} / \mathrm{mrad}^{2} / 0.1 \%$ BW has been obtained in the $\mathrm{keV}$ range this way at the Advanced Light Source. The critical frequency, above which the radiation begins to fall off exponentially, is $\omega_{\text {crit }}=3 \gamma^{2} \omega_{N R C}$, where $\gamma$ is the Lorentz parameter of the electrons, $\omega_{N R C}=e B / m \sim 10^{-4} \mathrm{eV}$ is the nonrelativistic cyclotron frequency for the magnetic field $B$ (typically $\sim 1 \mathrm{~T}$ ) in the bending magnet, $e$ is the electron charge, and $m$ is the electron mass. To generate hard $\mathrm{x}$-rays requires electrons in the $10-\mathrm{GeV}$ range. To improve the spectral brilliance, static undulator magnets are used to generate narrow-band $\mathrm{x}$-rays at a wavelength $\lambda_{x} \sim \lambda_{u} / \gamma^{2}$, where $\lambda_{u}$ is the period of the undulator. The properties of magnet materials restrict $\lambda_{u}$ to a few centimeters, so hard $\mathrm{x}$-rays require electrons in the $10-\mathrm{GeV}$ range. Spectral brightness in excess of $10^{19}$ photons $/ \mathrm{s} / \mathrm{mm}^{2} / \mathrm{mrad}^{2} / 0.1 \%$ BW in the $10-\mathrm{keV}$ range has been achieved this way at the Advanced Photon Source.

To avoid the GeV electrons required for conventional undulators, Compton backscatter of laser photons (sometimes called laser undulators) from a relativistic electron beam can be used. The x-ray photon energy is then $h v_{x}=4 \gamma^{2} h v_{L} \sim 5 \gamma^{2} \mathrm{eV}$ for a 1- $\mu \mathrm{m}$ laser, so hard x-rays can be generated using $10-50-\mathrm{MeV}$ electrons. The linewidth of the $\mathrm{x}$-rays is determined by the emittance of the electron beam, which causes the focused electrons to cross the axis at an angle. When the laser and the electron beam are optimally focused in the interaction region, the average spectral brilliance is found to be

$$
\bar{B}_{v} \sim \frac{\pi \sigma_{T}}{2 h e c^{2}} \frac{h v_{x}}{h v_{L}} \frac{\tau_{e}}{\tau_{L}+\tau_{e}} \hat{B}_{N} \bar{P}_{L}
$$

where $\sigma_{T}$ is the Thomson cross section, $h$ is Planck's constant, $c$ is the speed of light, $\tau_{e}$ is the electron pulse length, $\tau_{L}$ is the laser pulse length (typically about the same as the electron pulse length), $\hat{B}_{N}$ is the peak normalized brightness of the electron beam, and $\bar{P}_{L}$ is the average power 


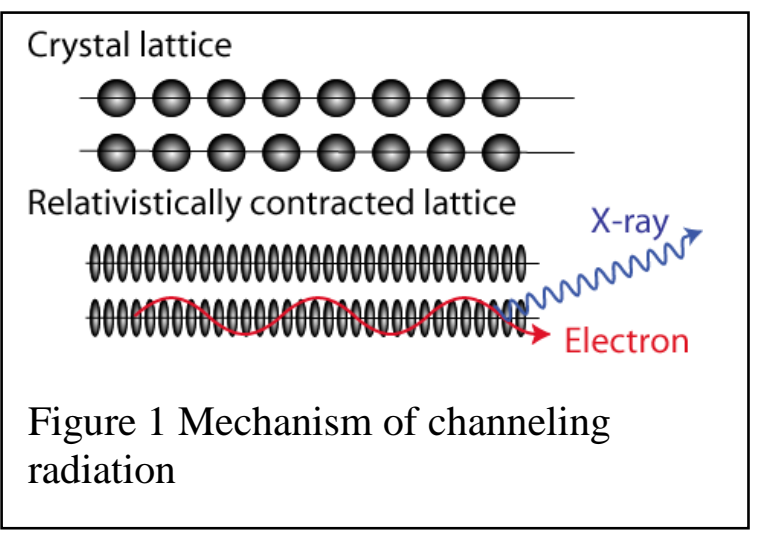

of the laser. The spectral brilliance will be reduced if the energy spread of the electrons is too large or if the intensity of the laser is high enough to shift the frequency of the backscattered photons. Although the above expression suggests that the spectral brilliance is independent of the electron-beam current, this is true only down to the current at which the broadening of the x-rays is dominated by the energy spread of the electron beam or the laser broadening, rather than the emittance. The only parameters in this expression are the peak brightness of the electron beam and the average laser power. For rf photoelectric injectors, the brightness is on the order of $10^{13} \mathrm{~A} / \mathrm{m}^{2}$-steradian, so to achieve a brilliance of $10^{12}$ photons $/ \mathrm{s}-\mathrm{mm}^{2}-\mathrm{mrad}^{2}-0.1 \% \mathrm{BW}$ in the $10-\mathrm{keV}$ range, an advance of two orders of magnitude beyond a conventional source, requires a laser average power of $1 \mathrm{~kW}$. This might be achieved by recirculating a laser beam of lower power. Alternatively, as discussed below, it may be possible to increase dramatically the electron-beam brightness at low peak current.

An interesting alternative to Compton backscatter is offered by channeling radiation. In a crystal, the ions in each crystal plane form a sheet of positive charge. When a relativistic electron travels through the crystal parallel to the crystal plane, Lorentz contraction increases the charge density by the factor $\gamma$ and the electron oscillates about the crystal plane in quantum states normal to the plane, as shown in Figure 1. Radiation from transitions between the quantum states is called channeling radiation. Channeling radiation was predicted theoretically by Kumakhov [i] in 1974, and observed experimentally by Terhune and Pantell [ii] in 1975.

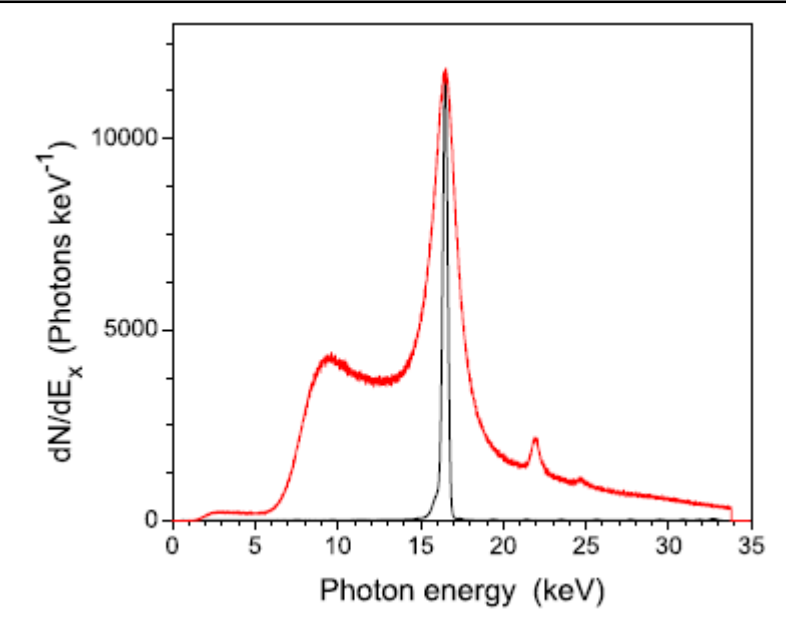

Figure 2 Observed spectrum of channeling radiation for transitions in (110) plane of diamond crystal at an electron energy of 14.6 $\mathrm{MeV}$ [Error! Bookmark not defined.]. Red: natural spectrum; black, monochromatized by Bragg reflection to remove the wings of the CR line and the Bremsstrahlung background.
Since then, there has been extensive theoretical and experimental investigation of channeling radiation, and theory and experiment are in good agreement.

The transverse forces experienced by an electron traveling along a crystal plane are comparable to those in a $10^{4}$-T magnetic undulator or a 1-TW laser undulator focused to a $10-\mu \mathrm{m}$ spot. The equivalent "undulator period" is on the order of 0.1 $\mu \mathrm{m}$. The coherence length of the crystal "undulator" is limited by scattering to something on the order of $1 \mu \mathrm{m}$, so the number of "undulator periods" in channeling radiation is on the order of 10 . The photon yield is more than 10 photons per electron at high energy $(\mathrm{GeV})$, but on the order of $10^{-4}$ photons per electron in the $\mathrm{x}$-ray region [iii]. The channeling-radiation peaks are typically an order of magnitude above the bremsstrahlung background [iv]. 


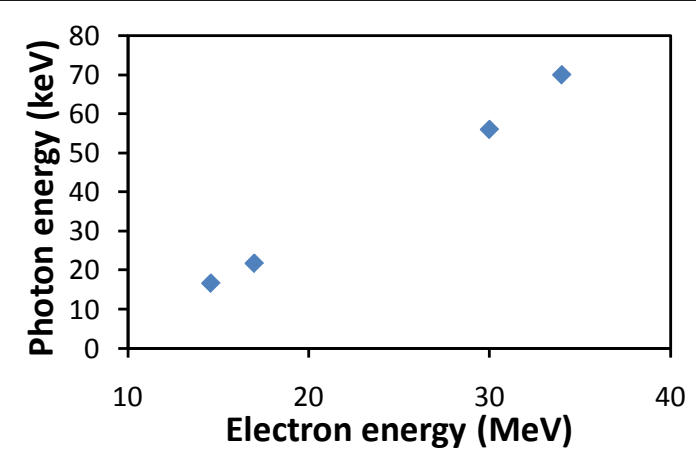

Figure 3 Photon energy of $1 \rightarrow 0$ transition in (110) plane of diamond, vs. electron energy [v].
The advantages of a channeling radiation source are clear. Compared with a conventional undulator, channeling radiation requires only a $40-\mathrm{MeV}$ electron beam, rather than a $10-\mathrm{GeV}$ beam to reach the hard $\mathrm{x}$-ray region. Compared with a laser undulator, a channeling radiation source comprises a small diamond chip rather than a complex laser system circulating a kilowatt of laser power.

For electrons channeling in diamond, the best available measurements are those of Azadegan [v]. A typical channeling

radiation spectrum is illustrated in Figure 2. The radiation is forward directed in a cone of angle $1 / \gamma$, and Doppler shifted by the factor $2 \gamma$. Including the Lorentz contraction of the crystal lattice, the photon energy scales roughly as $\gamma^{1.7}$, and spans the $\mathrm{x}$-ray and gamma-ray regions. For the $1 \rightarrow 0$ transition in (110) diamond, the photon energy may be tuned from 10 to $80 \mathrm{keV}$ by varying the electron energy from 10 to $40 \mathrm{MeV}$, as shown in Figure 3. For a 30-MeV electron incident on a 42.5-micron thick diamond crystal, the yield on the $1 \rightarrow 0$ transition corresponds to about 0.028 photons-steradian-keV in a line $3 \mathrm{keV}$ wide centered near $56 \mathrm{keV}$ [v]. In conventional units, this corresponds to $10^{-9}$ photons-mrad ${ }^{2}-0.1 \%$ BW per electron.

The spectral brilliance of the $\mathrm{x}$-radiation depends on how tightly the electron beam can be focused, and this depends on the emittance and the convergence angle of the beam. At the radiation source ELBE, in Dresden, a high-intensity channeling radiation source has been developed using a high-brightness electron beam incident on a diamond crystal [iii]. Diamond is the best material for this application owing to its high thermal conductivity [vi]. The normalized emittance of the beam after aperturing was $3 \mu \mathrm{m}$ rms at an average current of $100 \mu \mathrm{A}$ [vii], which corresponds to a peak brightness on the order of $10^{9} \mathrm{~A} / \mathrm{m}^{2}$-sterad. The beam was focused to a mm-size spot on the diamond. As much as $10^{11}$ photons/s were obtained in a $10 \%$ bandwidth, which corresponds roughly to an average spectral brilliance on

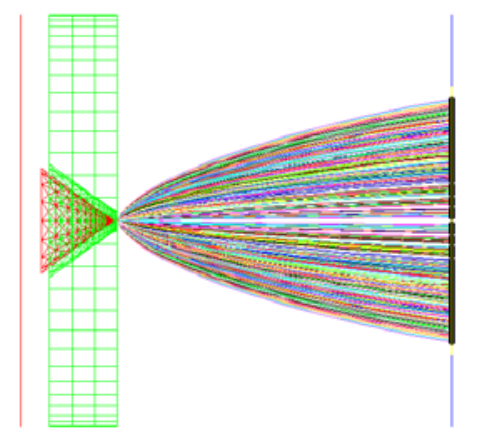

Figure 4. Simulation of a gated field emitter using CPO. the order of $10^{6}$ photons $/ \mathrm{s}-\mathrm{mm}^{2}-\mathrm{mrad}^{2}-0.1 \% \mathrm{BW}$.

The innovation that we propose is to use a single fieldemitting tip as the current source, in place of the gridded thermionic gun used at ELBE. To estimate the emittance from a single field-emitting tip, we have used the code CPO to simulate the emission from a diamond field emitter with a gated structure, as shown in Figure 4. A second gate can be used as a focusing electrode. The simulations yield a normalized emittance of $1.3 \mathrm{~nm}$, which is an improvement of three orders of magnitude over the ELBE thermionic gun. In experiments at Vanderbilt, we have observed more than $10 \mu \mathrm{A}$ average current from a single field-emitting tip [viii] with 
brightness approaching the quantum limit [ix]. The current was limited by damage to the anode, which was in close proximity. The ultimate performance of diamond tips has yet to be determined. By using a single tip it may be possible to improve the transverse brightness of the electron beam by as much as six orders of magnitude, with a corresponding improvement of the spectral brilliance of the x-ray beam. The absolute emittance of the beam from a single tip, extrapolated to $30 \mathrm{MeV}$, is $40 \mathrm{pm}$. If we focus this at the critical angle for channeling radiation in diamond (about $1 \mathrm{mrad}$ ), we get a spot diameter of $40 \mathrm{~nm}$; the spectral brilliance of the x-ray beam is then $10^{12}$ photons/s-mm $-\mathrm{mrad}^{2}-0.1 \% \mathrm{BW}$ at an average current of $200 \mathrm{nA}$.

Finally, the beam must be accelerated to high energy to create hard x-rays. At the present time, this acceleration would most like be staged. First, an rf gun would bunch and accelerate the beam to relativistic energies ( 3-5 MeV) sufficient for the beam to become relativistic. Calculations indicate that by biasing the gate electrode (Figure 4) with low-level rf in a combination of the first and third harmonics of the rf fundamental, the emission can be restricted to about 12 degrees centered at the optimum phase. The relativistic bunch would then be accelerated to its final energy by a conventional linac. The rf gun would play a crucial role in bunching the field-emitted beam while preserving the transverse beam quality. Another option would incorporate an rf gun cavity resonating at two harmonic frequencies. The phases and amplitudes of the respective electromagnetic field could then be selected to gate the field emission as illustrated in Fig. 5 without the need for a microfabricated gate electrode [x]. Such a technique could, in principle, provide field-emitted bunches with durations much shorter than the wavelength of the accelerating (fundamental) mode. Preserving the exquisite emittances produced by the tip after subsequent acceleration and manipulation will be challenging. Chromatic aberrations due to energy spread in the beam, emittance dilution due to nonlinearity in the rf fields, geometric aberrations in the electron beam transport lines, and collective effects will have to be thoroughly mitigated. Since such an electron beam source represents an order-of-magnitude level increase in electron beam quality, other effects which to date have been unnoticed, may also become important. One main concern, for instance, is the extent to which Coulomb collisions at low energies (Boersch effect) will contribute to phase space dilution [xi].

In the future, an x-ray source based on an rf accelerator could be made compact by using X-band linac technology. X-band linacs have undergone intense development for application to the Next Linear Collider and, more recently, for several x-ray FEL projects [xii]. Acceleration gradients in excess of $100 \mathrm{MV} / \mathrm{m}$ have now been achieved in several laboratories [xiii], so that a 40-MeV accelerator - capable of producing $85-\mathrm{keV} \mathrm{CR}$ photons - only requires $40 \mathrm{~cm}$ of acceleration. Further in the future, higher-frequency accelerators fabricated lithographically may make it possible to place the entire accelerator and x-ray source on a single chip [xiv]. 
Because the x-rays are produced by the interaction of the electron beam with the crystal, the maximum current of the electron beam is limited by heating of, and radiation damage to, the crystal. Measurements and computations show that for diamond at room temperature the effects of heating are acceptable up to a few mA of beam current, so this will not be a limitation even for $\mathrm{cw}$ operation [vi]. Measurements show that radiation damage becomes significant above a total beam fluence on the order of a few $\mathrm{C}$ per square centimeter. Thus, the $40-\mathrm{nm}$ focal spot is destroyed in about $100 \mu \mathrm{s}$. The crystal must be moved at about $1 \mathrm{~mm} / \mathrm{s}$, and the crystal is destroyed at the rate of 0.1 square millimeters per hour.

i M. A. Kumakhov, Phys. Lett. A 57, 17 (1976).

ii R. W. Terhune and R. H. Pantell, Appl. Phys. Lett 30, 265 (1977).

iii W. Wagner, B. Azadegan, M. Sobiella, J. Steiner, K. Zeil, and J. Pawelke, Nucl. Instrum. Meth. B 266, 327

(2008).

iv M. A. Kumakhov, International Conference on Charged and Neutral Particles Channeling Phenomena, edited by S. B. Dabagov, Proc. SPIE 5974, (SPIE, Bellingham, WA, 2005).

v B. Azadegan, Ph.D. thesis, Technischen Universität Dresden, 2007.

vi C. K. Gary, R. H. Pantell, M. Ozcan, M. A. Piestrup, and D. G. Boyers, J. Appl. Phys. 70, 2995 (1991).

vii W. Neubert, B. Azadegan, W. Enghardt, K. Heidel, J. Pawelke, W. Wagner, Nucl. Instrum. Meth. B 254, 319 (2006).

viii J. D. Jarvis, H. L. Andrews, C. A. Brau, B.-K. Choi, J. Davidson, W.-P. Kang, and Y.-M. Wong, J. Vac. Sci. Technol. B 27, 2264 (2009).

ix J. D. Jarvis, H. L. Andrews, B. Ivanov, C. L. Stewart, N. de Jonge, E. C. Heeres, W.-P. Kang, Y.-M. Wong, J. L. Davidson, and C. A. Brau, J. Appl. Phys. 108, 094322 (2010).

x J. W. Lewellen and J. Noonan, Phys. Rev ST Accel. Beams 8, 033502 (2005).

xi H. Boersh, Ziet. Physik, 139, 115-146 (1954).

xii T. Raubenheimer, presented at the Conference on the Application of Accelerators in Research in and Industry, Fort Worth, TX, August 8-13, 2010.

xiii F.Wang, C. Adolphsen, and C. Nantista, Phys. Rev. ST-AB 14, 010401 (2011).

xiv A. Nassiri, at al., Proc. Int. Electron Devices Meeting, Washington, DC, December, 1993. 